\title{
First Epileptic Seizure and Initial Diagnosis of Juvenile Myoclonus Epilepsy (JME) in a Transcranial Direct Current Stimulation (tDCS) Study- Ethical Analysis of a Clinical case
}

\author{
Anna Sierawska $(\mathbb{D} \cdot$ Vera Moliadze • Maike \\ Splittgerber • Annette Rogge • Michael Siniatchkin • \\ Alena Buyx \\ Received: 25 March 2020 / Accepted: 28 June 2020 / Published online: 1 July 2020 \\ (C) The Author(s) 2020, corrected publication 2021
}

\begin{abstract}
We discuss an epileptic incident in an undiagnosed 13-year old girl participating in a clinical study investigating the effects of transcranial direct current stimulation (tDCS) in healthy children and adolescents. This incident poses important research ethics questions with regard to study design, especially pertaining to screening and gaining informed consent. Potential benefits and problems of the incident also need to be considered. The ethical analysis of the case presented in this paper has been informed by an in-depth interview conducted after the incident with the child and the accompanying parent. We discuss the ethical implications of the epileptic incident, the need for improving screening procedures for studies with minors and for providing more effective communication. This case also
\end{abstract}

\footnotetext{
A. Sierawska $(\bowtie) \cdot$ A. Buyx

Institute for History and Ethics in Medicine, Medical School, Technical University of Munich, Ismaninger Strasse 22,

81675 Munich, Germany

e-mail: anna.sierawska@tum.de

V. Moliadze $\cdot$ M. Splittgerber

Institute of Medical Psychology and Medical Sociology,

University Medical Center Schleswig Holstein, Kiel University,

Kiel, Germany

A. Rogge

Division of Biomedical Ethics, Institute of Experimental

Medicine, Kiel University, Kiel, Germany

M. Siniatchkin

Clinic for Child and Adolescent Psychiatry and Psychotherapy,

Medical Center Bethel, Bielefeld, Germany
}

underscores the problem of undetected teenage epilepsy in neuropsychological clinical studies and the necessity of raising more awareness of this issue. Since research in tDCS is an active and expanding field, we conclude with providing some recommendation that could ensure that future research on tDCS, or other therapies and neurointerventions where there is a risk of triggering an epileptic seizure, take into account the specifics of teenage epilepsy and the need for more thorough provision of information during the process of gaining informed consent.

Keywords tDCS · Non-invasive brain stimulation . Paediatrics · Adverse event - Epilepsy · Research ethics

\section{Introduction}

Transcranial direct current brain stimulation (tDCS) is a brain stimulation technique where a weak current is applied between head-mounted electrodes in order to facilitate either depolarization or hyperpolarization of neurons $[1,2]$. There is an increasing interest in applying tDCS in treatment for various neurological and psychiatric disorders [3] also in children and adolescents $[4,5]$. TDCS is perceived as a well-tolerated technique and according to recent guidelines, no serious adverse effects have been directly linked to its application [6]. It was noted, however, that due to, e.g., lack of long-term studies, the promises of tDCS should be assessed with caution $[7,8]$. 
TDCS is an expanding field. The main challenges in development and application of tDCS in pediatrics involve very limited number of studies, and difficulties in translating and transferring the evidence from adult studies into pediatric populations [8]. Children are a vulnerable group, and thus any type of research, especially with novel technologies, comes with various ethical issues, e.g. capacity to make informed decisions, assessment of child's best interest and parental responsabilities with regard to child's research participation [9]. One of the ways of ensuring that those challenges are not overlooked and addressed in a timely fashion is the close cooperation between researchers and ethicists from the earliest stage of research. The reported case happened in a tDCS study OPTI-Stim study in the H2020-funded consortium STIPED (STIPED, Trial DRKS00008207). In STIPED, an ethics work package is part of the interdisciplinary consortium. Its role is to monitor risks during the project, and to assess the social attitudes towards the technique. Within this work package, we designed empirical studies that investigate views and experiences of children and their parents participating in the clinical tDCS study. In our research, we cooperate closely with the clinical study teams. Next to the empirical work, the monitoring task of the ethics work package involves ensuring the compliance with the research ethics guidelines and support in discussing unexpected ethical issues. Here, we present our process with regard to an adverse event that happened to a participant enrolled in a control group study. In this short report, we provide a preliminary ethical analysis of the incident, underlining the importance of our findings for all neuro-interventions, especially in pediatric populations.

The ethical analysis was prompted by the case of a 13-year-old subject who presented a first generalized tonic-clonic epileptic seizure (GTCS) in the week following her tDCS procedure [10]. The GTCS as well as myoclonic jerks on awakening and EEG abnormalities, were consistent with the diagnosis of Juvenile Myoclonic epilepsy (JME). It was established during the followup meeting with the study team that the participant had features of JME prior to the study and that these were not identified during the screening appointment. Hence, it is important to stress that the occurrence of the seizure might have been unrelated to the tDCS. However, by bringing this case and its implication to the attention of the academic community, we would like to highlight the importance of introducing more in-depth pre-study screening, particularly in pediatric populations, that could improve the inclusion and exclusion criteria for tDCS studies.

\section{Ethical Case Description and Analysis}

\section{Clinical Background}

The OPTI-Stim study aims to explore the effects of anodal tDCS over the left dorsolateral prefrontal cortex (IDLPFC) in healthy children. OptiStim focuses on two general objectives: 1) to characterize interaction between brain development and effects of tDCS on neuropsychological function and 2) to apply individual head modelling and electrical current estimation to guide individualized treatment with tDCS in different stages of development. OptiStim is a phase-I randomized double-blind sham-controlled crossover study with six measurement points and includes healthy subjects of different ages from 10 to 18 years old. Exclusion criteria, based on current guidelines [6], were pregnancy, history of migraine, unexplained loss of consciousness, or brain related injury, IQ $<80$, birth weight $<$ 2500 g., born before the 37th week of pregnancy, history or family history of epileptic seizures, history of other neurological, psychiatric or chronical internistic disorders, intake of central nervous system-effective medication, brain- or cardiac- pacemakers, or not removable metal head implants.

A 13-year-old girl was included in the OPTI-Stim clinical study. Five days after the second tDCS session, she was brought to the Children's Clinic by emergency ambulance due to a first GTCS. She was found in the garden of her house, breathing heavily followed by an epileptic seizure. A routine wake and sleep deprivation scalp EEG recording was performed on two occasions following the GTCS. The GTCS as well as myoclonic jerks on awakening and EEG abnormalities were strongly suggestive of a diagnosis of JME. Precipitating factors, including sleep deprivation, medication ingestion or concurrent illness, were excluded [10].

JME is a common genetically determined ("idiopathic") generalized epilepsy, which tends to present within the $10-25$ years age group. The incidence of epilepsy in children ranges between 41 and 187 cases per 10,000 [11]. The prevalence is consistently higher than the incidence and lies between 3.2-5.5 cases per 1000 in developed countries and 3.6-44 in underdeveloped 
countries. Only about one third of children with epilepsy can be assigned to a specific epilepsy syndrome and many cases remain unreported [11]. This uncertainty has implications for provision of information prior to signing the informated consent, as it makes it more difficult for the researchers and subsequently also the participants to assess the risk.

Recent neuroimaging studies [12] have suggested that JME may be characterized as a frontal lobe variant of a multi-regional, thalamocortical "network" epilepsy. Since tDCS was performed mostly over the frontal lobe in this subject who had undisclosed features of JME, it could be argued that tDCS could have produced reduction of seizure threshold by inducing regional change in brain network excitability. On the other hand, no epileptic abnormalities were captured in the study EEG recordings, which were of good technical quality [10].

Below we present findings from the ethics interview conducted with the participant who experienced the epileptic seizure and her mother and provide further ethical case analysis. The mother and daughter agreed to the publication of our findings.

\section{Ethics Interview and Analysis}

The GTCS rates as a serious adverse effect (SAE) in the study. It was reported to the local ethics committee at the University Medical Center Schleswig Holstein in Kiel [10]. Two months after the incident, an in-depth ethics interview was conducted with the participant and her mother. This interview aimed to explore participant experiences, expectations and worries with regard to the tDCS study and also discuss the seizure incident. Overall, both the participant and the mother had a good experience with the clinical study and were enthusiastic about the research participation. They considered tDCS as more beneficial (less harmful) when compared with medication for neuropediatric disorders, saying: "better [to treat] from the outside than from the inside". The discussion of the epileptic case uncovered that it was only communicated to the research team when the participant attended her third appointment. In spite of explaining the potential risks and exclusion criteria during screening, neither the participant nor the mother remembered to inform the study team immediately about the seizure. In the follow up meeting with the OPTI-Stim study team the participant discussed retrospectively that she experienced trembling and incidents that could have been classified as minor seizures.
However, it was only after the incident that this was fully investigated, hence it was not communicated during the screening interview and it could thus not serve as a reason to exclude the participant.

There are several ethical issues pertaining to the assessment and reporting of the epileptic seizure in this case. Firstly, it could be argued that initial screening for the study, which followed tDCS screening guidelines for adults is not detailed enough for pediatric populations, as it only explores the possibility of a diagnosis of epilepsy or other neurological disorders, without exploring the symptoms. Specific questions that would explore the possibility of epilepsy more in-depth were not included in the screening questionnaire. Reporting this case might contribute to changing the screening guidelines accordingly. Secondly, the reported case shows that neither the mother nor the participant paid enough attention during the informed consent procedure to remember that any type of epilepsy should be reported to the research team immediately. Finally, regarding the risk-benefit assessment of study participation in pediatric tDCS it should be noted that even though the causal link between the seizure and tDCS cannot be confirmed, the occurrence of the seizure had some beneficial aspects: it led to further tests and eventually a correct diagnosis was made and appropriate treatment was ordered.

\section{Discussion and Recommendations}

Analysis of this case has revealed that the information provided during the screening process was insufficient and that a juvenile epilepsy might get overlooked during the initial research stage. In the expanding field of pediatric tDCS, it is an important finding, especially in the light of limited tDCS evidence and safety guidelines in pediatric population $[8,13]$. With this report we would like to point out to the importance of adapting the pre-study screening guidelines accordingly. This is particularly relevant since it been shown that current research can present selective bias in reporting adverse side effects in tDCS trials [14]. Adverse effects are also often described inadequately [15]. Our case also illustrates the difficulties stemming from ambiguity in the classification of an undiagnosed epilepsy as either a side effect or an incidental finding. A more in-depth screening interview and additional, targeted questions about epilepsy symptoms could be helpful measures to timely 
identify the risk of epilepsy. The study team as a result of the incident added a question on occurrence of twitching/trembling in the limbs, especially in the morning hours, to their screening questionnaire. We also recommend adding further questions such as "Have [you/the child] ever experienced moments where you have lost track of your surroundings, then suddenly snapped back to alertness?" to be considered for future research. Whereas a tailored (more detailed) EEG during screening could enhance the diagnostics, in subjects without any risk detected during the questioning phase, it would be time-consuming and could also lead to more initial costs.

The case also highlights the problem of potential overestimation of parental and participants' understanding, in spite of researchers following the guidelines set by the ethics committee. While study participants should not be worried unnecessarily, it is important that they are made fully aware about potential side effects, about uncertainties regarding potential risks, and the importance of immediately reporting any unusual medical situation to the research team. This could be ensured e.g. by advising researchers to ask more control questions during the informed consent process or using more interactive approaches [13], and also by altering appropriately the study questionnaires to effectively report the adverse effects [14].

\section{Conclusion}

A serious adverse event, namely an epileptic seizure, occurred in a healthy teenage participant in a tDCS study, leading to a subsequent diagnosis of epilepsy. The participant and the mother expressed that even if the stimulation had contributed to the seizure, this would have been for the best, as it led to receiving a correct diagnosis and appropriate treatment could be initiated. Although it cannot be established with full certainty what the exacts factors were that contributed to the seizure, it is important that the screening protocol is updated accordingly for pediatric populations, especially adding more detailed information regarding epilepsy symptoms, such as trembling. Accurate phrasing should be used so the parents and the children can answer targeted screening questions correctly. The case analysis shows also the importance of more in-depth provision of information and a more patient-centered approach during the screening process. Such changes should be supported by ethics committees and other regulatory bodies.

With the rising interest in various forms of "noninvasive" stimulations [16], it is extremely important that participants are made aware of potential side effects and that more awareness in general of undetected pediatric epilepsy is raised during screening. At the same time, this should be balanced with the need to avoid scaring, medicalizing and over-diagnosing participants unnecessarily. A detailed anamnesis by a neuropediatrician might be needed where the screening interview reveals risks.

Funding Information Open access funding enabled and organized by Projekt DEAL. This project has received funding from the European Union's Horizon 2020 research and innovation program under grant agreement no. 731827 . This text reflects only the authors' views and the Commission is not liable for any use that may be made of the information contained therein.

\section{Compliance with Ethical Standards}

Competing Interests All authors declare no competing interests.

Ethics Approval Approval was obtained from the local ethics committee: Ethics Committee University Medical Center Schleswig-Holstein, approval number D 449/17.

Open Access This article is licensed under a Creative Commons Attribution 4.0 International License, which permits use, sharing, adaptation, distribution and reproduction in any medium or format, as long as you give appropriate credit to the original author(s) and the source, provide a link to the Creative Commons licence, and indicate if changes were made. The images or other third party material in this article are included in the article's Creative Commons licence, unless indicated otherwise in a credit line to the material. If material is not included in the article's Creative Commons licence and your intended use is not permitted by statutory regulation or exceeds the permitted use, you will need to obtain permission directly from the copyright holder. To view a copy of this licence, visit http://creativecommons.org/licenses/by/4.0/.

\section{References}

1. Nitsche, M.A., and W. Paulus. 2001. Sustained excitability elevations induced by transcranial DC motor cortex stimulation in humans. Neurology 57 (10): 1899-1901.

2. Nitsche, M.A., and W. Paulus. 2000. Excitability changes induced in the human motor cortex by weak transcranial direct current stimulation. The Journal of physiology 527 (Pt 3): $633-639$. 
3. Utz, K.S., V. Dimova, K. Oppenlander, and G. Kerkhoff. 2010. Electrified minds: Transcranial direct current stimulation (tDCS) and galvanic vestibular stimulation (GVS) as methods of non-invasive brain stimulation in neuropsychology-a review of current data and future implications. Neuropsychologia. 48 (10): 2789-2810.

4. Palm, U., F.M. Segmiller, A.N. Epple, F.J. Freisleder, N. Koutsouleris, G. Schulte-Korne, et al. 2016. Transcranial direct current stimulation in children and adolescents: a comprehensive review. Journal of neural transmission (Vienna, Austria : 1996) 123 (10): 1219-1234.

5. Finisguerra, A., R. Borgatti, and C. Urgesi. 2019. Noninvasive brain stimulation for the rehabilitation of children and adolescents with neurodevelopmental disorders: A systematic review. Frontiers in Psychology 10: 135.

6. Antal, A., I. Alekseichuk, M. Bikson, J. Brockmoller, A.R. Brunoni, R. Chen, et al. 2017. Low intensity transcranial electric stimulation: Safety, ethical, legal regulatory and application guidelines. Clinical neurophysiology : official journal of the International Federation of Clinical Neurophysiology. 128 (9): 1774-1809.

7. Sierawska, A., A. Prehn-Kristensen, V. Moliadze, K. Krauel, R. Nowak, C.M. Freitag, et al. 2019. Unmet Needs in Children With Attention Deficit Hyperactivity Disorder-Can Transcranial Direct Current Stimulation Fill the Gap? Promises and Ethical Challenges. Frontiers in psychiatry 10: 334.

8. Davis, N.J. 2014. Transcranial stimulation of the developing brain: A plea for extreme caution. Frontiers in human neuroscience. 8(AUG).

9. Nuffield Council on Bioethics. 2015. Children and clinical research: Ethical issues. In: Bioethics NCo, editor. London.
10. Splittgerber, M., Japaridze, N., Sierawska, A., Gimenez, S., Nowak, R., Siniatchkin, M., et al. 2019. First generalized tonic clonic seizure in the context of pediatric tDCS - A case report. Neurophysiol Clin.

11. Camfield, P., and C. Camfield. 2015. Incidence, prevalence and aetiology of seizures and epilepsy in children. Epileptic Disorders 17 (2): 117-123.

12. Wang, Y., I.S. Berglund, M. Uppman, and T.Q. Li. 2019. Juvenile myoclonic epilepsy has hyper dynamic functional connectivity in the dorsolateral frontal cortex. NeuroImage Clinicale 21: 101604.

13. Kadam, R.A. 2017. Informed consent process: A step further towards making it meaningful! Perspectives in Clinical Research 8 (3): 107-112.

14. Brunoni, A.R., J. Amadera, B. Berbel, M.S. Volz, B.G. Rizzerio, and F. Fregni. 2011. A systematic review on reporting and assessment of adverse effects associated with transcranial direct current stimulation. The international journal of neuropsychopharmacology. 14 (8): 1133-1145.

15. Aparicio, L.V.M., F. Guarienti, L.B. Razza, A.F. Carvalho, F. Fregni, and A.R. Brunoni. 2016. A systematic review on the acceptability and tolerability of Transcranial direct current stimulation treatment in neuropsychiatry trials. Brain Stimulation 9 (5): 671-681.

16. Wexler, A. 2017. The social context of "do-it-yourself" brain stimulation: Neurohackers, biohackers, and Lifehackers. Frontiers in Human Neuroscience 11: 224.

Publisher's Note Springer Nature remains neutral with regard to jurisdictional claims in published maps and institutional affiliations. 